\title{
Cholesterol or Fat Rich Diets Accelerate Natural Age-Decline on Adult Hippocampal Neurogenesis and Have an Impact in Memory and Like-Anxiety Behavior
}

\author{
Perla Leal-Galicia, María Carmen Sánchez-Torres, Marco Antonio Meraz-Ríos \\ Departamento de Biomedicina Molecular, Centro de Investigación y de Estudios Avanzados del InstitutoPolitécnico Nacional, \\ Instituto Politécnico Nacional, México City, México \\ Email:mmeraz@cinvestav.mx
}

How to cite this paper: Leal-Galicia, P., Sánchez-Torres, M.C. and Meraz-Ríos, M.A. (2019) Cholesterol or Fat Rich Diets Accelerate Natural Age-Decline on Adult Hippocampal Neurogenesis and Have an Impact in Memory and Like-Anxiety Behavior. Advances in Bioscience and Biotechnology, 10, 331-345.

https://doi.org/10.4236/abb.2019.1010026

Received: August 26, 2019

Accepted: October 21, 2019

Published: October 24, 2019

Copyright $\odot 2019$ by author(s) and Scientific Research Publishing Inc. This work is licensed under the Creative Commons Attribution International License (CC BY 4.0).

http://creativecommons.org/licenses/by/4.0/

\begin{abstract}
Diet is an important health factor and it has been recently associated with neurodegenerative diseases and cognitive decline. Here it was investigated the effect of fatty acid or cholesterol rich diets with the possible acceleration of the biological decline in adult hippocampal neurogenesis associated with aging in middle-age rats, and its impact on anxiety and memory function. It was found that a diet of 10 weeks with saturated fatty acids and cholesterol has a detrimental effect on memory function, exerts like-anxiety behavior and diminishes the presence of new generated neurons in the hippocampus in six months old rats.
\end{abstract}

\section{Keywords}

High-Fat Diet, High-Cholesterol Diet, Adult Hippocampal Neurogenesis, Memory, Like-Anxiety Behavior

\section{Introduction}

Neurodegenerative diseases and obesity are some of the biggest challenges to deal in our days. It has been recently proposed a link between these two phenomena being obesity and diet habits, a trigger in neurodegeneration onset; however, the mechanism underlying such relationship has not been well established yet. It has been suggested that components of the so called "Western diet" such as saturated fatty-acids and cholesterol can have an important participation in this phenomenon. Epidemiological studies have shown that Japanese people liv- 
ing in their ethnic hometown have a lower prevalence of Alzheimer Disease (AD) than those living in the USA [1]. A similar observation occurs in Nigerian Africans compared with African Americans [2]. These observations strongly suggest that environmental factors, such as dietary habits and life style in industrialized cities can contribute to the development of alterations in the brain. It is important to investigate the participation of diet-induced elevation of lipids in brain and its relationship with brain alterations. This is relevant since the brain is an organ with high amount of lipids as well as essential fatty acids, which are obtained from the diet. Such molecules gained from the diet have an important participation in brain functioning [3]. On the other hand, it is very well known that very little of systemic cholesterol can cross the blood-brain barrier (BBB), and brain cholesterol is produced in situ and its participation on brain functioning is crucial. Cholesterol is involved in processes such as myelination, membrane structure and cell signaling [4]. Epidemiological studies demonstrated a link between consumption of diets rich in saturated fat and cognitive alterations [5] [6] [7]. In addition, studies carried out in animal models have demonstrated that a diet rich in fat can have a negative impact on learning and memory function, [8]. Diets rich in fat or cholesterol induced elevation in serum cholesterol and triglycerides, and produced alterations on hippocampal morphology and deficits in memory performance in middle-age rats [9] [10]. These studies support a detrimental effect on hippocampal function induced by fats and cholesterol rich diets, and it raises the question whether the detrimental effect exerted in the hippocampus by fat or cholesterol intake can have an impact on adult hippocampal neurogenesis. The adult hippocampal neurogenesis (AHN) is a highly specialized type of neuronal plasticity that occurs in the hippocampal dentate gyrus and it has been described to be regulated in response to different stimuli, allowing the animal to adapt to environmental changes [11] [12] [13]. AHN has been associated with memory function and its detriment results in deficits in memory flexibility [14] and alterations in short-term memory [15]. It has been reported that some food components can modulate some parts of the neurogenesis process (for a review see [16]). For instance, a diet rich in fat can decrease the number of new generated hippocampal cells in young male rats after 4 weeks of treatment [17]. Mice fed for 17 weeks with a high-fat diet exhibit a reduced number of immature neurons in the hippocampal dentate gyrus in juvenile individual, producing alterations on relational memory flexibility and spatial discrimination learning, showing that adolescence is a critical period for the detrimental impact of a high fat diet in adult hippocampal neurogenesis. Middle age is a critical period for adult hippocampal neurogenesis as it is known that age is a natural down-regulator of adult hippocampal neurogenesis, and from 6 months old onwards, the adult hippocampal neurogenesis decreases [15] [18] [19]. This work is focused in evaluating if rats fed with a saturated fatty acid diet (FAD) or a cholesterol enriched diet (CED) at age where natural decrease of AHN occurs, could enhance the diminishment of hippocampal neurogenesis, worsening these natural phenomena. The present work evaluates if the con- 
sumption of these types of diets by a period of 10 weeks could accelerate the neurogenesis decline associated with aging, and identify if these diets could contribute to an increase in the cognitive decline associated with age. The Novel Object Recognition Memory Test was carried out in order to evaluate the impact of the dietary treatments in memory performance. Since mood disorders have been associated with detrimental cognitive abilities in elderly, here it was investigated if fat or cholesterol intake could have an impact on anxiety-like behaviors. It was found that extended consumption of diets rich in fat or cholesterol is contributing to enhancement of cognitive decline. The effect is associated to fat metabolism and aging throughout the diminishment of AHN with a lower number of new generated cells, changes in the complexity of the newly generated cells, and affected the functionality of the individuals with changes in memory ability and expression of anxiety-like behavior.

\section{Methods}

\subsection{Animals}

All procedures involving animal subjects were carried out according to the institutional regulations CINVESTAV-IPN guided for the care and use of laboratory animals. Protocol approval was obtained from the Institutional Animal Care and Use Committee (Protocol number 0081-14). In order to perform the study, 25 female 5 months old Wistar rats at the beginning of the experiment were employed. Rats were obtained from the Institutional Animal Facility (IAF) and distributed in 3 groups. Animals were kept in controlled light, air and humidity conditions and received food and water ad libitum. Rats were randomly assigned to the experimental groups as follows: Control diet $(\mathrm{n}=8)$, fatty acid $\operatorname{diet}(n=8)$, cholesterol enriched diet $(n=9)$. Animals were fed by 10 weeks with the different diets, which components are described in Table 1. Body weight was weekly registered. All the institutional and international regulations were followed in order to avoid animals suffering and reduce the number of animals employed in the study.

\subsection{Blood Samples and Lipids Profile}

At week 0 , all the animals were taken to the experimental room and were anesthetized with $5 \%$ isoflurane for induction and $3 \%$ for maintenance in an anesthesia chamber (Vetequip Impac6). Once the animals felt under the effect of the anesthesia, the low paws were shaved and blood was collected with a cannula from the femoral vein. Blood samples were kept in Eppendorf tube with heparin and store at room temperature. After this procedure, animals were returned to the home cages, blood samples were centrifuged and plasma was collected for further analysis. The parameters studied were: total cholesterol, triglycerides, high density lipids (HDL), and low density lipids (LDL). The samples were sent to a clinical veterinarian laboratory for its analysis. The animals had $24 \mathrm{hr}$ of fasting before collecting the blood samples in order to avoid immediate effects of diet. 
Table 1. Nutritional composition of pellets.

\begin{tabular}{ccl}
\hline \multirow{2}{*}{ Group } & \multicolumn{2}{c}{ Table Column Head } \\
\cline { 2 - 3 } & \multicolumn{1}{c}{ Diet } & \multicolumn{1}{c}{ Composition } \\
\hline $\begin{array}{c}\text { Control diet } \\
\text { (Ctrl) }\end{array}$ & $\begin{array}{c}\text { Standard laboratory } \\
\text { diet pellets }\end{array}$ & \multicolumn{1}{c}{$10 \%$ Kcal fat } \\
$\begin{array}{c}\text { Fatty-acid diet } \\
\text { (FAD) }\end{array}$ & Fatty-acid enriched pellets & $\begin{array}{l}\text { Diet induced obesity (DIO) composition, 60 } \\
\text { Kcal\% Fat. (Casein 200g, 3 g L-cysteine) (Jackson } \\
\text { Laboratory D12492). }\end{array}$ \\
Cholesterol & Cholesterol enriched pellets \\
enriched (CED) & $\begin{array}{l}\text { Diet-induced Atherosclero- } \\
\text { sis/Hypercholesterolemia in Rodent Models, Diet } \\
\text { 3, 40 Kcal\% Fat, 1.25\% Cholesterol (Jackson } \\
\text { Laboratory, D12108). }\end{array}$ \\
\hline
\end{tabular}

\subsection{BrdU Injections}

By the 6th week of treatment, the animals received a single dose of 5-Bromo-2'Deoxyuridine (BrdU) (50 mg/Kg bodyweight, B5002_SIGMA) in order to label proliferating cells in the hippocampal dentated gyrus.

\subsection{Elevated Plus Maze}

In order to analyze possible emotional modulation induced by diet, the rats were tested with the elevated plus maze (EPM) test, by the 3rd week after BrdU injection. This is a widely used anxiety test that explores behaviors phylogenetically determined. It takes advantage of the natural aversion of rats to open/high spaces and test how animals can cope with a level stressor [20]. The test was performed as reported in Leal-Galicia et al., [15]. The apparatus is an elevated surface with cross shape where two arms are enclosed by walls and two are open. The animal is placed in the center of the cross facing the enclosed arm and is left there to freely explore for $300 \mathrm{sec}$. Low anxiety animals will spend more time in the open arms. The number of crossing between the open and enclosed arms, as well as the time spend exploring the open arms were measured.

\subsection{Novel Object Recognition Memory}

In order to measure the impact of our dietary treatments on memory function, the Novel Object Recognition Memory (NORM) was carried out by the 30th day after BrdU injection. This test is based on the natural rat behavior of exploring new items. It has the advantage of requiring non-previous training and does not induce stress in the animals. In addition, comprises exposition to novelty, which can involve new hippocampal generated cells participation. NORM measures the ability of rodents to discriminate between the familiarities of an object previously encountered versus a new object. Rodents tend to be curious and explore novel objects, the animals will recognize, more rapidly, the known object and will spend more time exploring the novel object [21]. The tests were carried out accordingly to what was previously reported in Leal-Galicia et al., [15]. Briefly, the test has three phases: habituation, acquisition and recognition. There is a $24 \mathrm{hr}$ 
interval between phases. Rats were move individually into the experimental room and carefully collocated in the center of an empty plastic box $(39 \mathrm{~cm}-55$ $\mathrm{cm}-65 \mathrm{~cm}$ ) for $5 \mathrm{~min}$ each (habituation phase). After $5 \mathrm{~min}$, rats were carefully taken back to the housing room. For the acquisition phase, rats were placed in the same box as in the habituation phase but containing two equidistant glass objects, one with cylindrical shape and the other one with rhomboid shape. Both have non-biological relevancy and were equally attractive. The time spent exploring the objects was recorded. After $5 \mathrm{~min}$, rats were carefully taken back to the housing room and the objects cleaned-up with a solution of $10 \%$ ethanol, $5 \%$ dextran in order to avoid any odor references. Recognition phase was carried out $24 \mathrm{hr}$ later. In this phase, rats were exposed with two different objects than those used in the acquisition phase. One with same shape and the other one completely different. Time exploring the known and the novel object was registered. Object recognition was evaluated as exploratory index calculated by dividing the total time exploring the novel object $(\mathrm{NO})$ by the total time exploring the $\mathrm{NO}$ plus the total time exploring the familiar object (FO) multiplied by $100: \mathrm{NO} /(\mathrm{NO}$ + FO) $\times 100$. Preference for an object corresponds to $>50 \%$ index [22].

\subsection{Tissue Preparation}

In order to analyze the effect of FAD or CED intake on hippocampal adult neurogenesis, $24 \mathrm{hr}$ after the memory test the animals were deeply anesthetized with sodic pentobarbital and transcardially perfused with $0.9 \%$ saline. Sub-epidermal fat from left low paw was collected in order to quantify fat gaining. Brains were obtained, and post-fixed in a fixative solution consisting of $4 \%$ formaldehyde, in

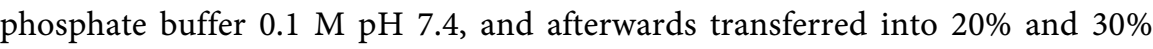
sucrose (24 hr each). Thereafter each brain was cut into $40 \mu \mathrm{m}$ serial coronal sections and kept free floating in a cryoprotectant solution (25\% ethylene glycol, $25 \%$ glycerol and 50\% $0.1 \mathrm{M}$ phosphate buffer; v/v) $4^{\circ} \mathrm{C}$. After that, one of every 6th section was collected to process by immunohistochemistry for cell proliferation and cell fate analysis. The cell markers analyzed were the neuronal migration protein doublecortin (DCX) for quantify number of newly generated neurons, NeuN/BrdU to quantify the number of newly generated mature cells and BrdU to measure cell proliferation. Briefly, free-floating sections were carefully washed $2 \mathrm{X}$ PBS in order to remove the cryoprotectant solution. For BrdU and DCX staining, the slices were incubated for 30 minutes with a solution containing $0.6 \% \mathrm{H}_{2} \mathrm{O}_{2}$ in PBS, to block endogenous peroxidases and then rinsed 3 times with TBS for 10 minutes each and 2 times with $0.9 \% \mathrm{NaCl}$ and then incubated 30 minutes in $2.5 \mathrm{HCl}$ at $37^{\circ} \mathrm{C}$. Tissues were rinsed 4 times in PBS and then transferred to the blocking solution containing $10 \%$ horse serum and $0.2 \%$ Triton $\mathrm{X}-100$ in $\mathrm{PBS}$ for $1 \mathrm{hr}$ at room temperature. Incubation with the primary antibody (Anti-NeuN antibody [1B7]-Neuronal Marker ABCAM (ab104224)1:500, SIGMA) or anti-goat DCX polyclonalanty body (Santa Cruz Biotechnology, Santa Cruz, CA, USA)1:250, diluted in a solution containing 3\% Horse serum 
and $0.2 \%$ Triton X-100 in PBS overnight at $4^{\circ} \mathrm{C}$. Samples were rinsed 3 times with PBS, 10 minutes each and incubated 2 hours with the secondary antibody (anti-mouse IgG or anti-goat Vector, Laboratories, Burlingame, CA). Slices were rinsed 3 times with $\mathrm{PBS}$ and $\mathrm{ABC}$ reagent was applied for $1 \mathrm{~h}$, followed by peroxidase detection for approximately $5 \mathrm{~min}$ using diaminobenzidine as chromogen $\left(0.25 \mathrm{mg} / \mathrm{ml}\right.$ diaminobenzidine, $0.01 \% \mathrm{H}_{2} \mathrm{O}_{2}, 0.04 \% \mathrm{NiCl}$, Vector Labs). For the double immunofluorescence, the sections were processed as was described for BrdU and the endogenous peroxidase elimination step was eliminated, in addition, the secondary antibody was coupled to a fluorochrome for confocal analysis. Ending the BrdU staining, the staining for NeuN was performed (Rabbit Anti-NeuN antibody, ABN78, Merck). Negative controls consisted of eliminating primary antibodies in the procedure.

\subsection{Cell Fate Analysis}

In order to quantify the number of BrdU and DCX positive cells in the sub-granular zone and in the granular cell layer of the dentate gyrus, fluorescent cells throughout the entire rostro-caudal extent of the hippocampus were counted employing a conventional confocal microscope. The optical dissector method was employed according to previously described in Kempermann et al., [23]. Briefly, one of each 6 sections was collected to be processed by free floating immunohistochemistry against BrdU and DCX. After carrying out the immunostaining (as was described before), the sections were mounted in coverslips and every cell stained for BrdU or DCX was exhaustively counted in every section. The total number of BrdU positive cells per animal was obtained after multiplied by 50 the number of positive counted cells.

\subsection{Statistical Analyses}

The statistical analyses were performed with GraphPad Prism 6. Due the behavioral data has not normal distribution, data was analyzed with Kruskal-Wallis test followed by Dunn's comparison multiple test, the data related to physiological measurements was analyzed with factorial analyses of variance (ANOVA) followed by Bonferroni post hoc test, where appropriate. Statistical significance is highlighted with a symbol when $\mathrm{p} \leq 0.05 ; 2$ symbols when $\mathrm{p} \leq 0.01 ; 3$ symbols when $\mathrm{p} \leq 0.001 ; 4$ symbols when $\mathrm{p} \leq 0.0001$. ${ }^{\star}$ refers compared to control, \& refers compared to group FAD. All values are given as means \pm SEM, with an $n=$ 8 to 9 animals in each condition. For the relationship between body weight and exploration index, the gained weight per animal compared with its score in the exploration index was plot.

\section{Results}

\subsection{FAD Induces an Increase in Bodyweight}

FAD induces an increase in bodyweight in female rats from week 4 of treatment onwards, in comparison with control and CED animals and such differences are 
maintained until the end of the treatment $(\mathrm{F}(10,88)=4.217, \mathrm{p} \leq 0.0001)\left({ }^{\circ} \mathrm{C}\right)$. In addition, induces a rising in epidermal fat weight compared with the control and CED treatment $(\mathrm{F}(2,22)=3.576, \mathrm{p}=0.0452)($ Figure $1(\mathrm{~b}))$.

\subsection{FAD and CED Induce Alterations in Lipids Processing}

Animals fed with the CED show a maintained significant increase in the levels of total cholesterol in plasma, which was very high at the end of the treatment $(F(2$, $20)=35.76, \mathrm{p} \leq 0.0001$ group significance deemed as \#) (Figure 2 (a) deemed as \#). This type of diet also induces a significant increase in LDL and triglyceride levels $(\mathrm{F}(2,23)=11.31, \mathrm{p}=0.0004$ group significance deemed as $\#)$ and $(\mathrm{F}(2,20)$ $=35.76, \mathrm{p} \leq 0.0001$ ) (Figure $2(\mathrm{~d})$ and Figure $2(\mathrm{~b})$ group significance deemed as \#) respectively. The group fed with FAD shows a highly significant rise in the levels of total cholesterol in plasma by the third week of treatment. However, at the end of the experiment the levels of cholesterol were similar to those observed in the control group $(\mathrm{F}(2,20)=35.76, \mathrm{p} \leq 0.0001$ group significance deemed as $\&)$. In addition, this diet also induces an increase on LDL levels by the third week $(\mathrm{F}(2,21)=4.1777, \mathrm{p}=0.0297$ group significance signaled as $\&)$, but does not induces significant changes on blood triglyceride levels. It was observed in the control group a maintained increase in total cholesterol and LDL levels but this is lower than those observed in the CED group $(F(2,21)=9.730, p \leq 0.0010$ group significance deemed as ${ }^{\star}$ ).

\subsection{Cholesterol Diet Induces Anxiety-Like Behaviors and FAD and CED Induce a Short-Term Memory Deficit}

EPM is a widely employed test to evaluate the ability of rodents to cope with a lower stressor and take advantage of the natural exploratory behavior of the rats,

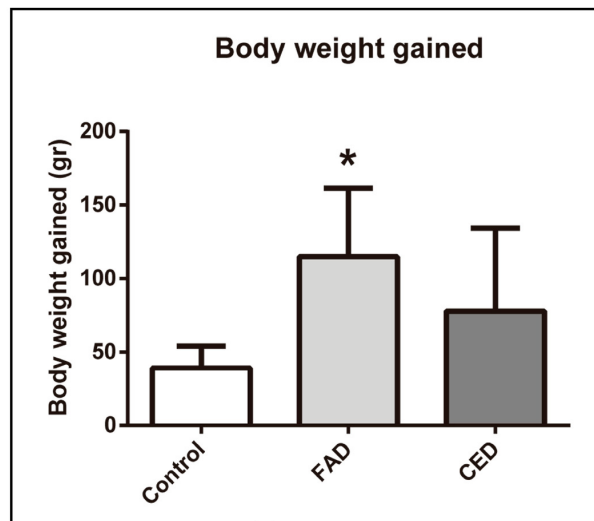

(a)

\section{Sub-epidermal fat weight}

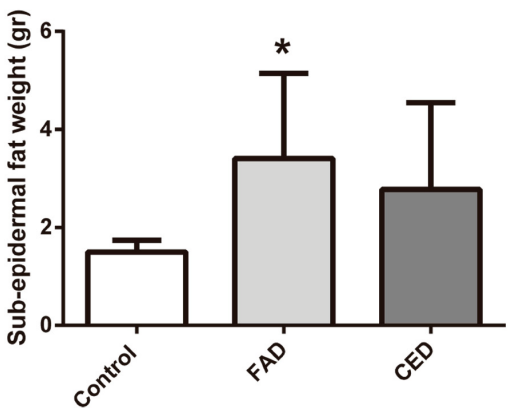

(b)

Figure 1. Bodyweight gained and sub-epidermal fat increase in animals fed with CED or FAD. (a) FAD produces a significant increase in bodyweight of rats fed with these type of diet by 10 weeks compared with the animals in control group (deemed as ${ }^{*}$ ) (b) Rats fed with FAD had higher volume of sub-epidermal fat in the law paws, compared with the animals in the control group (deemed $\mathrm{s}^{*}$ ) ${ }^{*}$ ) Compared control group. Bonferroni post hoc test after ANOVA $\mathrm{p}<0.05$. 


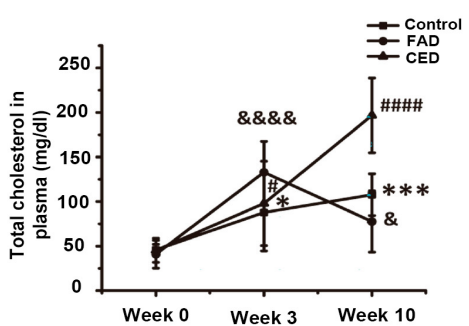

(a)

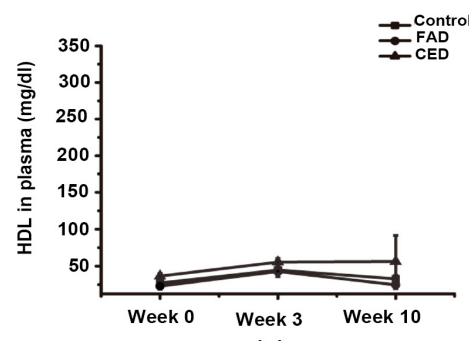

(c)

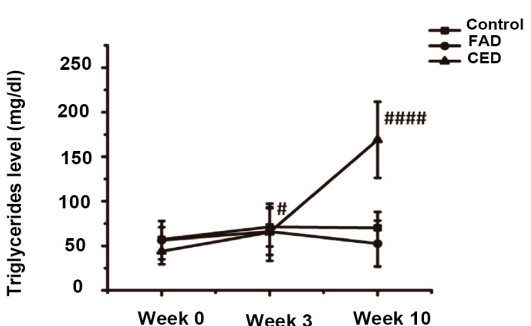

(b)

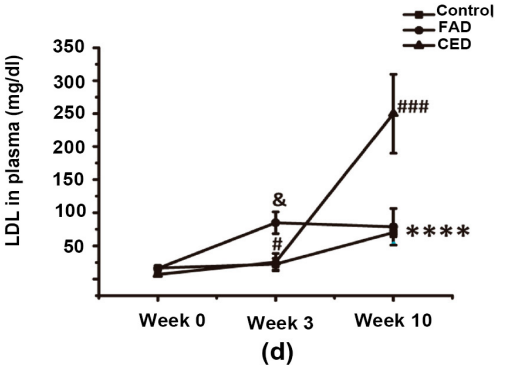

(d)

Figure 2. Dysregulation of lipids processing after 10 weeks of FAD or CED intake. (a) CED consumption induces an increase in levels of total cholesterol in plasma from 3 weeks and it reaches very high levels by the week 10, compared with control group (deemed as \#). It is observed as well in the FAD group (deemed as \&). (b) Levels of triglycerides show a significant increase from 3 rd week in plasma samples of animals fed with CED (deemed as \#), this effect is not exerted by consumption of FAD. (c) There is no evidence of modulation of HDL levels by consumption of FAD or CED in plasma samples of rats fed with these diets. (d) There is a significant increase of LDL levels in plasma of rats fed with CED and FAD from the 3rd weeks and it shows a rise by the 10th week. (\#) Comparing CED with control group. (\#, equals to $\mathrm{p}<0.05$, \#\#\# equals to $\mathrm{p}<0.0001$ ). (\&) FAD compared with control group) ( $\&$, equals to $\mathrm{p}<0.05$, \&\&\&\& equals to $\mathrm{p}<$ 0.0001. Bonferroni post hoc test after ANOVA $\mathrm{p}<0.05)$.

and does not require a previous training program. It is known that rats with better deal with the stressor, in this case an open elevated space, will explore more time in the open arms portion. Female rats fed 10 weeks with CED spent less time exploring in the open arms region compared with control group or FAD (Kruskal-Wallis test followed by Dunn's comparison multiple test $\alpha=0.05$ ), which suggest an anxiety-like behavior (Figure 3(a)). EPM is a test that requires from the animal an appropriate mobility in order to be properly performed, the number of crossings between arms (both open and enclosed) was measured, in order to discard that the differences observed in the number of crossings were not related to mobility problems and avoid false results due to aging and overweight. There were no significant differences between the locomotion reported as the number of crossings between arms (Figure 3(b)). The animals moved the same among arms but the animals in the CED group prefer the enclosed arms, which supports the idea that CED treated animals show a higher anxiety-behavior. In other words, the behavior observed is not a lack of locomotion but a preference for exploring the "more-safe" enclosed portion. 


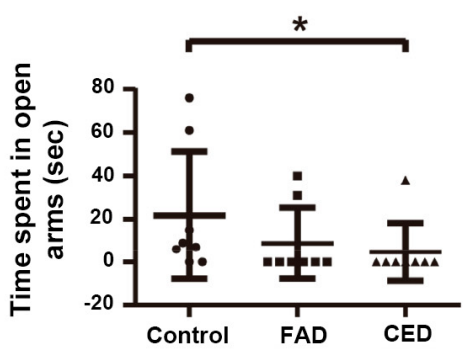

(a)

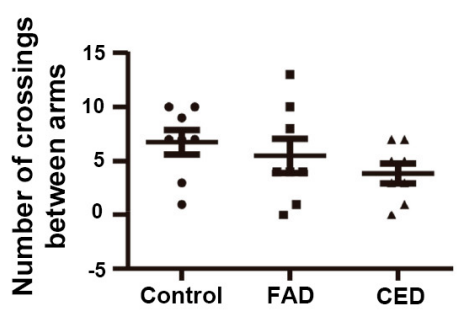

(b)

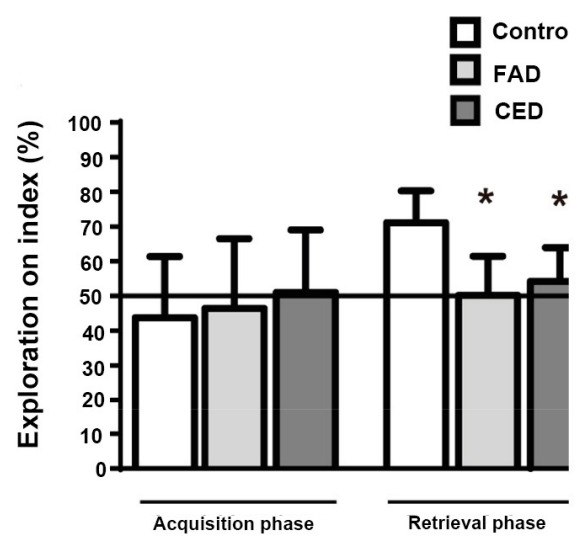

(c)

Figure 3. Consumption of FAD or CED promotes alteration in behavior. (a) CED intake induces a decrease in the time spend in open arms in the EPM but not in locomotion measured as number of crossing between arms, which suggest that the consumption of CED induces anxiety-like behavior (deemed as * compared with control group); (b) Fed female rats by 10 weeks with a CED or a FAD decreases the ability to discriminate a novel object versus an already known, reported as exploration index, it suggest an alteration in memory (deemed as * compared with control group. For EPM Kruskal-Wallis test followed by Dunn's comparison multiple test $\alpha=0.05$, for NORM, Bonferroni post hoc test after ANOVA $\mathrm{p}<0.05$ ).

NORM evaluates the ability of rats to differentiate between a known object and a new one, taking advantage of the natural preference of the rats to explore novelty objects. An exploration index close to or below to $50 \%$ indicates that the animals explored equally the known and the novel object, suggesting that rats did not remember that they were exposed to the known object. Rats fed with a FAD had an exploratory index of 50.23\%, which indicates that the rats did spend the same time exploring the known and the novel object. Rats fed with CED also had an exploration index close to 50\% (54.32\%) (Figure 3(b)) which indicate that some of them explored the new object slightly more than the known one, but still smaller than the control group which had a $71.08 \%$ of exploration index. These results reveal that FAD and CED induce a significant detriment in memory performance.

\subsection{FAD and CED Decrease Survival and Differentiation of Hippocampal New Generated Cells}

All animals received an i.p. injection of BrdU, an analogue of thymidine that allows to label cells under S-phase and is used to identify hippocampal neurons under proliferation. In order to label the activation of the new neurons, the BrdU injection was carried out 30 days before the memory test, which is the time where the new generated neurons are able to fire and can be identified by the Activity-Regulated Cytoskeleton-associated protein (ARC) [24]. Rats fed with CED show a strong significant decrease on the number of BrdU positive cells compared with Control group $(\mathrm{F}(2,18)=17.05, \mathrm{p} \leq 0.0001$, Figure $4(\mathrm{a}))$. A significant diminishment is observed as well in FAD group compared with Control group $(\mathrm{F}(2,18)=17.05, \mathrm{p} \leq 0.0001$, Figure $4(\mathrm{a}))$. What can be concluded from 


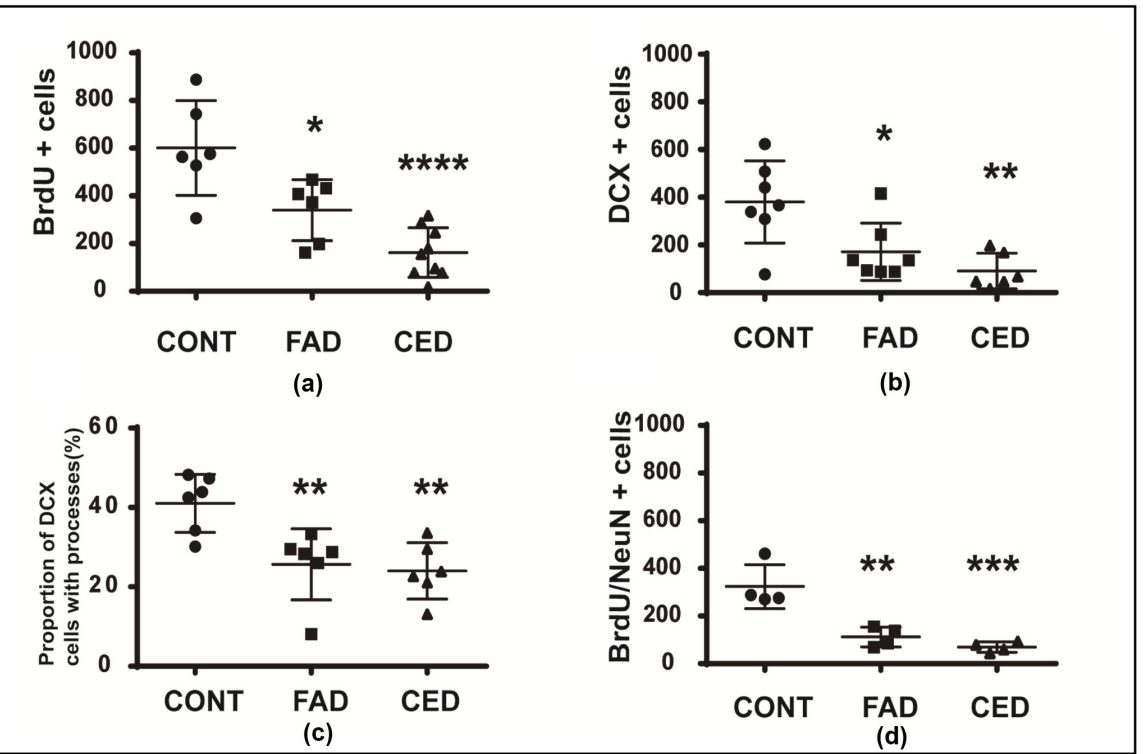

Figure 4. FAD and CED reduces the number of survival and differentiation of new born hippocampal neurons. (a) Total number of BrdU positive cells exhibits a decrease in the hippocampal dentate gyrus of rats fed with FAD and CED for 10 weeks (deemed as * compared with control group); (b) The hippocampus of rats fed with FAD and CED have significantly less number of DCX positive cells compared with the animals in control groups (deemed as ${ }^{\star}$ ); (c) as well as less number of DCX cells with large processes (deemed as * compared with control group); (d) Dentate gyrus of rats fed with FAD and CED have a decrease in the pool of new generated cells that become fully mature neurons compared with control animals ( ${ }^{*}$, equals to $\mathrm{p}<0.05$, ${ }^{* *}$ equals to $\mathrm{p}<0.01$, ${ }^{* *}$ equals to $\mathrm{p}$ $<0.001{ }^{* * *}$ equals to $\mathrm{p}<0.0001$. Bonferroni post hoc test after ANOVA $\mathrm{p}<0.05$ ).

these data, is that CED and FAD induce a down-regulation on neuronal hippocampal proliferating cells.

Thereafter, the number of cells under maturation process were quantified, for that, it was employed the DCX marker. DCX is a cytoskeleton protein expressed in new neurons under migration [25]. It has been proposed that this subset of immature cells is important for information processing [26]. It was observed that both groups CED and FAD had a significant decrease on the number of DCX positive cells compared with control group and CED showed the lower values. The CED treated group have a significant less DCX positive cells compared with the FAD group $(F(2,20)=6.374, \mathrm{p}=0.0072)$ (Figure $4(\mathrm{~b}))$. On the other hand, in addition of the decrease on DCX cell number, there were changes in cell complexity, measured as the percentage of cells with developed processes $(\mathrm{F}(2,15)=8.619, \mathrm{p}=0.0032$, Figure $4(\mathrm{c}))$. It was observed that the dietary treatments affect cell differentiation and the cholesterol diet has a stronger effect. Particularly in the number of newly generated cells that went into the fully mature stage. NeuN is a nuclear cell factor present in mature granular cells, and double staining of BrdU/NeuN shows that feeding middle-age rats with a FAD or CED significantly reduce the number of new generated hippocampal cells that become mature $(F(2,12)=31.37, p \leq 0.0001$, Figure $4(d))$. 
Regarding ARC expression, it was exhaustively searched for it all over the hippocampal axis and there were found only some spread cells in few animals (data not shown) therefore it can be concluded that with the memory test performed here and for the time that the tissue was obtained, there are no detectable ARC cells.

\section{Discussion}

We have previously reported that consumption of a diet rich in saturated fatty-acid or cholesterol induces a maintained increase on bodyweight onwards 9 weeks in young male rats. In the current work, it was observed a clear bodyweight gaining in female middle-age rats especially in those fed with FAD. These rats accumulate more sub-epidermal fat in the law paws compared with controls. Fat accumulation is a reference for obesity. Obesity has been implicated as a trigger of a wide range of neuropsychiatric disorders such as depression (for a review see [27]) and there is more evidence that obesity could be a risk factor for developing dementia [28]. Here it was studied the effect of FAD or a CED in middle-age female rats on the age-depended decline of adult hippocampal neurogenesis (AHN). Lindqvist and colleagues have shown previously that male rats fed for 4 weeks with a diet with $42 \%$ of fat (coconut butter and corn oil) exhibit a decrease on the number of BrdU positive cells with non-increase in adipose tissue but an increase in corticosterone levels, which is a stress hormone. Those results occur in male rats but not in females and interestingly; there were no differences in the number of mature newly generated cells [17]. On the contrary, the results presented here show that female rats fed for 10 weeks with diet $60 \%$ fat (Casein $200 \mathrm{~g}, 3 \mathrm{~g}$ L-cysteine) do increase adipose tissue and diminish cell proliferation and the number of differentiated and fully maturation cells in the hippocampus. The controversial data can be due to the differences in the type of fat employed in both studies (coconut butter and corn oil versus Casein and L-cysteine) or also because of the rat strain. The results presented here, and Lindqvist show that the consumption of a diet rich in fat can be detrimental for brain plasticity due to the diminishment of the adult hippocampal neurogenesis, and the intake of a high fat diet has a detrimental impact on the entire hippocampal region. Calvo-Ochoa et al., [29] reported that a 7 days diet supplemented with $10 \%$ lard accompanied with drinking water containing $20 \%$ sucrose decreases hippocampal weight, reduces the dendritic arborization in CA1 as well as decreases the levels of the microtubule-associate protein 2 (MAP-2), reduces the number of the dendritic spines and synaptophysin content in CA1. In other words, consumption of a diet high in fat has a detrimental impact on hippocampal morphology. Boitard et al. [30] reported a decrease in the number of DCX cells in 3 weeks old mice fed with a diet composed by $24 \%$ ( $45 \% \mathrm{kcal})$ fat, mostly saturated fat from lard, $41 \%(35 \% \mathrm{kcal})$ carbohydrate, $20 \%(17.5 \% \mathrm{kcal})$ sucrose and also shows alterations in relational memory evaluated with radial maze. In addition, the authors did not find significant differences in the number of DCX positive cells in 12 weeks old mice fed for the same time with a high-fat 
diet. In contrast, here is observed significant differences in the FAD group compared with the control group observing a diminishment in DCX positive cells, as well as BrdU positive cells and fully mature cells. It is possible that the differences could be due in the diet content as well as the animal strain. The results presented here, describe for the first time the changes in three steps of cells progression in the new generated hippocampal cells: proliferation, differentiation and maturation, during a critical age period where hippocampal neurogenesis shows a natural decrease. The data presented in the current study, demonstrate that dietary habits could have an impact on hippocampal neurogenesis and further analysis is necessary to investigate the molecular mechanisms involved in such diminishment. Finally, the detrimental impact of ingesting high amounts of cholesterol and fatty acids in adults could alter hippocampal neurogenesis and produce a significant increase in cognitive decline.

Here it was also found that both diets have a detrimental impact on memory with an exploratory index lower than $50 \%$, which means that the animals equally explored the known and the novel object, suggesting that the animals did not remember the known object. These results support the previous reports where several types of memory can be diminished when rats or mice are fed with a FAD or a CED [8] [9] [10]. AHN is an important component of learning and memory function [14] [15] [31] [32] and there is a decrease associated with aging, but little is known regarding who modulates this natural diminishment [15] [18] [19]. The consumption of a FAD or CED induces a lower number of BrdU positive cells compared with the control. In addition, these animals have less number of cells committed to neuronal linage and present a poor cell complexity, related to a concomitant impact on memory performance. These animals do not show the ability to remember information previously presented. This alteration is closely related to hippocampus functioning and is widely known that this cognitive failure is associated with age cognitive decline and is one of the first features observed in dementia cognitive alterations. Interestingly, it was observed that animals fed with CED show anxiety-like behaviors. It may resemble mood instability reported in middle cognitive impairment and early stages of dementia. The results in the current investigation, show that consumption of FAD and CED contribute to the appearance of behavioral features related to dementia in middle age rats. It could be linked to dyslipidemia and the decrease of the amount of new neurons in the hippocampus and an alteration in the complexity of the newborn cells. The data reported in this study, even though is descriptive, supports the idea that intake of diets rich in fat or cholesterol are risk factors for cognitive decline and can contribute to the development of dementia. Further investigation should be conducted in order to elucidate the molecular mechanism of the phenomena described here.

\section{Acknowledgements}

The authors thank to Dr. Jorge Fernández Hernández Head of Animal Facility CINVESTAV-Zacatenco for his technical advice, as well as Julio Cesar Ramírez, 
the MSc María del Pilar Figueroa Corona and Benjamín E. Chávez-Álvarez for the invaluable technical support and Ana Valencia-Olvera from University of Illinois at Chicago, for the language revision and the helpful comments on the paper.

\section{Conflicts of Interest}

The authors declare no conflicts of interest regarding the publication of this paper.

\section{References}

[1] White, L., Petrovitch, H., Ross, G.W., Masaki, K.H., Abbott, R.D., Teng, E.L., Rodriguez, B.L., Blanchette, P.L., Havlik, R.J., Wergowske, G., Chiu, D., Foley, D.J., Murdaugh, C. and Curb, J.D. (1996) Prevalence of Dementia in Older Japanese-American Men in Hawaii. Journal of the American Medical Association, 276, 955-960. https://doi.org/10.1001/jama.1996.03540120033030

[2] Hendrie, H.C., Osuntokun, B.O., Hall, K.S., Ogunniyi, A.O., Hui, S.L., Unverzagt, F.W., Gureje, O., Rodenberg, C.A., Baiyewu, O. and Musick, B.S. (1995) Prevalence of Alzheimer's Disease and Dementia in Two Communities: Nigerian Africans and African Americans. American Journal of Psychiatry, 152, 1485-1492.

https://doi.org/10.1176/ajp.152.10.1485

[3] Vance, J.E., Karten, B. and Hayashi, H. (2006) Lipid Dynamics in Neurons. Biochemical Society Transactions, 34, 399-403. https://doi.org/10.1042/BST0340399

[4] Vance, J.E., Hayashi, H. and Karten, B. (2005) Cholesterol Homeostasis in Neurons and Glial Cells. Seminars in Cell \& Developmental Biology, 16, 193-212. https://doi.org/10.1016/j.semcdb.2005.01.005

[5] Kalmijn, S., Launer, L.J., Ott, A., Witteman, J.J., Hofman, A. and Breteler, M.M. (1997) Dietary Fat Intake and the Risk of Incident Dementia in the Rotterdam Study. Annals of Neurology, 42, 776-782. https://doi.org/10.1002/ana.410420514

[6] Requejo, A.M., Ortega, R.M., Robles, F., Navia, B., Faci, M. and Aparicio, A. (2003) Influence of Nutrition on Cognitive Function in a Group of Elderly, Independently Living People. European Journal of Clinical Nutrition, 57, 54-57. https://doi.org/10.1038/sj.ejcn.1601816

[7] Morris, M.C., Evans, D.A., Bienias, J.L., Tangney, C.C. and Wilson, R.S. (2004) Dietary Fat Intake and 6-Year Cognitive Change in an Older Biracial Community Population. Neurology, 62, 1573-1579. https://doi.org/10.1212/01.WNL.0000123250.82849.B6

[8] Winocur, G. and Greenwood, C.E. (2005) Studies of the Effects of High Fat Diets on Cognitive Function in a Rat Model. Neurobiology of Aging, 26, 46-49. https://doi.org/10.1016/j.neurobiolaging.2005.09.003

[9] Granholm, A.C., Bimonte-Nelson, H.A., Moore, A.B., Nelson, M.E., Freeman, L.R. and Sambamurti, K. (2008) Effects of a Saturated Fat and High Cholesterol Diet on Memory and Hippocampal Morphology in the Middle-Aged Rat. Journal of Alzheimer's Disease, 14, 133-145. https://doi.org/10.3233/JAD-2008-14202

[10] Linnea, R., Freeman, V., Haley-Zitlin, Stevens, Ch. and Granholm, A.Ch. (2011) Diet-Induced Effects on Neuronal and Glial Elements in the Middle-Aged Rat Hippocampus. Nutritional Neuroscience, 14, 32-44.

https://doi.org/10.1179/174313211X12966635733358 
[11] Kempermann, G. (2002) Why New Neurons? Possible Functions for Adult Hippocampal Neurogenesis. Journal of Neuroscience, 22, 635-638. https://doi.org/10.1523/JNEUROSCI.22-03-00635.2002

[12] Fabel, K., Wolf, S.A., Ehninger, D., Babu, H., Leal-Galicia, P. and Kempermann, G. (2009) Additive Effects of Physical Exercise and Environmental Enrichment on Adult Hippocampal Neurogenesis in Mice. Frontiers of Neuroscience, 10, 50. https://doi.org/10.3389/neuro.22.002.2009

[13] Kempermann, G., Fabel, K., Ehninger, D., Babu, H., Leal-Galicia, P., Garthe, A. and Wolf, S.A. (2010) Why and How Physical Activity Promotes Experience-Induced Brain Plasticity. Frontiers of Neuroscience, 8, 189. https://doi.org/10.3389/fnins.2010.00189

[14] Garthe, A., Roeder, I. and Kempermann, G. (2016) Mice in an Enriched Environment Learn More Flexibly Because of Adult Hippocampal Neurogenesis. Hippocampus, 26, 261-271. https://doi.org/10.1002/hipo.22520

[15] Leal-Galicia, P., Castañeda-Bueno, M., Quiroz-Baez, R. and Arias, C. (2008) Long-Term Exposure to Environmental Enrichment since Youth Prevents Recognition Memory Decline and Increase Synaptic Plasticity Markers in Aging. Neurobiology of Learning and Memory, 90, 511. https://doi.org/10.1016/j.nlm.2008.07.005

[16] Zainuddin, M.S. and Thuret, S. (2012) Nutrition, Adult Hippocampal Neurogenesis and Mental Health. British Medical Bulletin, 103, 89-114. https://doi.org/10.1093/bmb/lds021

[17] Lindqvist, A., Mohapel, P., Bouter, B., Frielingsdorf, H., Pizzo, D., Bundin, P. and Erlanson-Albertsson, C. (2006) High-Fat Diet Impairs Hippocampal Neurogenesis in Male Rats. European Journal of Neurology, 13, 1385-1388. https://doi.org/10.1111/j.1468-1331.2006.01500.x

[18] Kuhn, H.G., Dickinson-Anson, H. and Gage, F.H. (1996) Neurogenesis in the Dentate Gyrus of the Adult Rat: Age-Related Decrease of Neuronal Progenitor Proliferation. Journal of Neuroscience, 15, 2027-2033. https://doi.org/10.1523/JNEUROSCI.16-06-02027.1996

[19] Kronenberg, G., Bick-Sander, A., Bunk, E., Wolf, C., Ehninger, D. and Kempermann, G. (2006) Physical Exercise Prevents Age-Related Decline in Precursor Cell Activity in the Mouse Dentate Gyrus. Neurobiology of Aging, 27, 1505-1513. https://doi.org/10.1016/j.neurobiolaging.2005.09.016

[20] Lister, G.G. (1990) Ethologically-Based Animal Models of Anxiety Disorders. Pharmacology and Therapeutics, 46, 321-340. https://doi.org/10.1016/0163-7258(90)90021-S

[21] Ennaceur, A. and Delacour, J. (1998) A New One-Trial Test for Neurobiological Studies of Memory in Rats. 1: Behavioral Data. Behavioral Brain Research, 1, 47-59. https://doi.org/10.1016/0166-4328(88)90157-X

[22] Clark, R.E., Kuczenski, R. and Segal, D.S. (2007) Escalating Dose, Multiple Binge Methamphetamine Regimen Does Not Impair Recognition Memory in Rats. Synapse, 61, 515-522. https://doi.org/10.1002/syn.20397

[23] Kempermann, G., Gast, D., Kronenberg, G., Yamaguchi, M. and Gage, F.H. (2003) Early Determination and Long-Term Persistence of Adult-Generated New Neurons in the Hippocampus of Mice. Development, 130, 391-399. https://doi.org/10.1242/dev.00203

[24] Sandoval, C.J., Martínez-Claros, M., Bello-Medina, P.C., Pérez, O. and Ramírez-Amaya, V. (2011) When Are New Hippocampal Neurons, Born in the 
Adult Brain, Integrated into the Network That Processes Spatial Information? PLoS ONE, 6, e17689. https://doi.org/10.1371/journal.pone.0017689

[25] Couillard-Despres, S., Winner, B., Schaubeck, S., Aigner, R., Vroemen, M., Weidner, N., Bogdahn, U., Winkler, J. Kuhn, H.G. and Aigner, L. (2005) Doublecortin Expression Levels in Adult Brain Reflect Neurogenesis. European Journal of Neuroscience, 21, 1-14. https://doi.org/10.1111/j.1460-9568.2004.03813.x

[26] Piatti, V.C., Davies-Sala, M.G., Espósito, M.S., Mongiat, L.A., Trinchero, M.F. and Schinder, A.F. (2017) The Timing for Neuronal Maturation in the Adult Hippocampus Is Modulated by Local Network Activity. Journal of Neuroscience, 25, 7715-7728. https://doi.org/10.1523/JNEUROSCI.1380-11.2011

[27] Rajan, T.M. and Menon, V. (2017) Psychiatric Disorders and Obesity: A Review of Association Studies. Journal of Postgraduate Medicine, 63, 182-190. https://doi.org/10.4103/jpgm.JPGM_712_16

[28] Xu, W.L., Atti, A.R., Gatz, M., Pedersen, N.L., Johansson, B. and Fratiglioni, L. (2011) Midlife Overweight and Obesity Increase Late-Life Dementia Risk. A Population-Based Twin Study. Neurology, 76, 1568-1574. https://doi.org/10.1212/WNL.0b013e3182190d09

[29] Calvo-Ochoa, E., Hernández-Ortega, K., Ferrera, P., Morimoto, S. and Arias, C. (2014) Short-Term High-Fat-and-Fructose Feeding Produces Insulin Signaling Alterations Accompanied by Neurite and Synaptic Reduction and Astroglial Activation in the Rat Hippocampus. Journal of Cerebral Blood Flow Metabolism, 34, 1001-1008. https://doi.org/10.1038/jcbfm.2014.48

[30] Boitard, C., Etchamendy, N., Sauvant, J., Aubert, A., Tronel, S., Marighetto, A., Layé, S. and Ferreira, G. (2012) Juvenile, But Not Adult Exposure to High-Fat Diet Impairs Relational Memory and Hippocampal Neurogenesis in Mice. Hippocampus, 22, 2095-2100. https://doi.org/10.1002/hipo.22032

[31] Dupret, D., Fabre, A., Döbrössy, M.D., Panatier, A., Rodríguez, J.J., Lamarque, S., Lemaire, V., Oliet, S.H., Piazza, P.V. and Abrous, D.N. (2007) Spatial Learning Depends on Both the Addition and Removal of New Hippocampal Neurons. PLoS $O N E$, 5, e214. https://doi.org/10.1371/journal.pbio.0050214

[32] Dupret, D., Revest, J.M., Koehl, M., Ichas, F., De Giorgi, F., Costet, P., Abrous, D.N. and Piazza, P.V. (2008) Spatial Relational Memory Requires Hippocampal Adult Neurogenesis. PLoS ONE, 9, e1959. https://doi.org/10.1371/journal.pone.0001959 